\title{
Soft micromachines run like clockwork
}

Micromachines can now be made from entirely biocompatible materials owing to a new photolithographic additive manufacturing method. As reported in Science Robotics, this technique was used to create a remotely controlled, implantable device for the on-demand, localized drug delivery in a bone cancer model.

Conventional microelectromechanical systems (MEMS) are constructed from non-biocompatible materials. Although there is great promise in the use of micromachines in medicine, this is currently held back by the manufacturing challenge of creating biocompatible and remotely controlled devices. "We saw a large opportunity to build a new class of implantable devices if these manufacturing challenges could be solved. Because biological materials are soft, they cannot be processed in the same way as silicon or metal, and so we had to develop new manufacturing techniques," explains Samuel Sia, who led the study.

The new technique produced intricate 3D structures with movable components made from polyethylene glycol-based hydrogels. "We have developed a method to make very small devices not from silicon or metal, but from biological materials," says Sia, whose group has previously worked on the microscale patterning of biomaterials, but until now was not able form complex 3D devices. This development also represents an advance over existing $3 \mathrm{D}$ printing technologies, which can produce microstructured biomaterials, although not with moving parts as required for MEMS.

The technique relies on finely controllable vertical adjustment, which is accomplished using a micrometer screw gauge as a stepper. This permits UV light-initiated polymerization of thin hydrogel layers with predetermined thickness, whereby a photomask defines the micropattern of each layer. Two other salient features of the method are the selective lift-off and bonding of adjacent hydrogel layers. Accordingly, a previously released layer can later be rebonded to the top of a micropatterned layer(s) to form complex 3D structures that include not only movable components, but also reservoirs. The researchers guided magnetic beads into particular reservoirs under a magnetic field, rendering the devices highly responsive to magnetism.

The team first fabricated a range of micromachines that demonstrated both linear and rotary motion. Among several examples, the most elegant was a Geneva drive - a gear mechanism (comprising a driving gear and a driven gear) used in the watchmaking industry to translate continuous rotation to precise intermittent movement. This design was enabled by the use of hydrogel components of complementary shapes but with different mechanical strengths. Specifically, a stiff gel formed the support, exterior and moving gears, whereas soft 'posts' that deform under external force sit between the spokes of the gear; this deformation allows the driven gear to rotate when a force is applied. In the absence of magnetic actuation, the posts do not deform and remain upright to hold the gear in place.

Each of the six spokes of the driven gear contained a reservoir. Five of these reservoirs were filled with the cancer drug, doxorubicin,

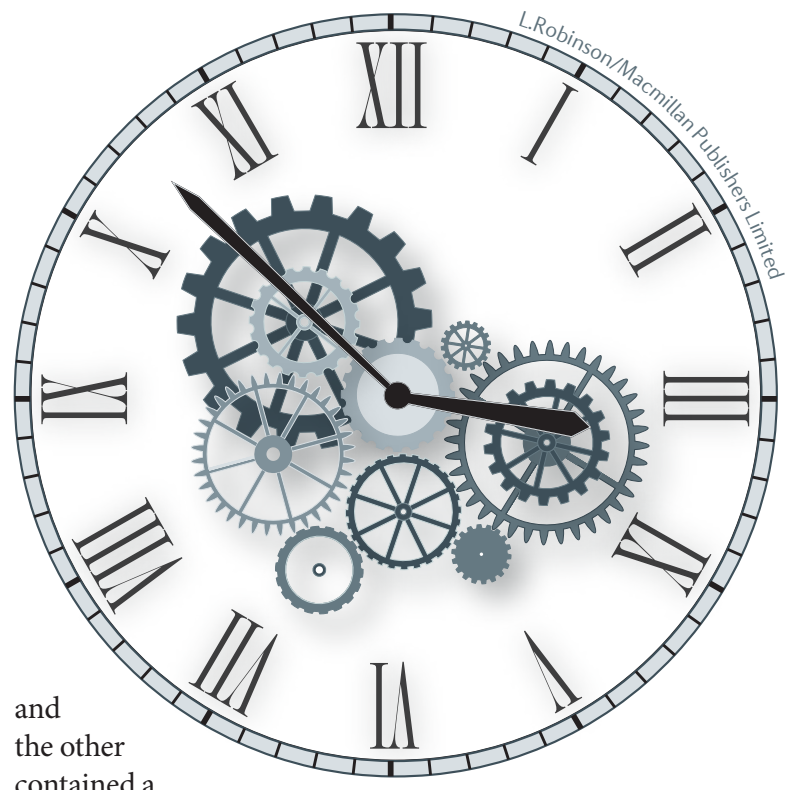

contained a

fluorescent agent to

allow the actuation mechanism to be followed in vivo. The magnetic particles were confined to the driving gear, which, in a magnetic field, applied sufficient force to rotate the driven gear by one position (that is, by $60^{\circ}$ ). This aligned the next reservoir with an aperture, through which the drug was released. The researchers tested the device in vivo in a mouse osteosarcoma model, delivering just $10 \%$ of a standard therapeutic dose. The localized administration resulted in enhanced tumour necrosis and reduced cardiotoxicity compared with the much higher systemic dose. Moreover, the pulsatile nature of this device is an advantage over existing, continuous-release hydrogel-based delivery systems.

In future work, "we are further exploring the drug delivery application and are developing different versions of the device to tackle different classes of medical problems," notes Sia. The ultimate goal is for such wirelessly controlled microdevices to deliver drugs for a range of diseases and to assist in the repair of damaged organs. More generally, the new fabrication method has important implications for the future design of soft robots and micromachines.

Adam Brotchie

ORIGINAL ARTICLE Chin S. Y. et al. Additive manufacturing of hydrogel-based materials for next-generation implantable medical devices. Science Robotics http://dx.doi.org/10.1126/ scirobotics.aah6451 (2017)

\section{4 \\ We have developed a method to make very small devices not from silicon or metal, but from biological materials}

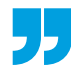

